\title{
Understanding Preferences for Search System Interfaces and the Role of Emotive Forces: A Research Challenge
}

\author{
Steven J. Bell
}

\begin{abstract}
Information professionals have observed that Web-based search interfaces simplify database searching and are preferred over other interfaces. A further anecdotal observation is that their features, in comparison to other interfaces, enhance the quality of search sessions, resulting in better outcomes. One explanation is that Web interfaces ease the negative emotive forces searchers experience with other interfaces. This paper examines the challenges in designing a research method to test the validity of these assumptions and observations. Although Web interfaces may provide a more supportive search setting, particularly for less experienced searchers, there is no evidence they contribute to better search outcomes.
\end{abstract}

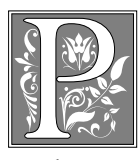

rofessional and end-user searchers of commercial online databases have more interface options today than at any time in the recent past. The three primary interface options are Windows clients for PCs or Macs, Web clients, and Telnet or DOS clients. Windows and Web clients are characterized by their point-and-click, graphical user interface (GUI), although Windows clients typically offer greater functionality than Web clients, whereas Telnet or DOS clients are character-based systems. Having more options means more confusion about choices. The PC client may offer the greatest level of search functionality, but it brings the burden of distributing client software to the user population and of offering an update mechanism each time a new upgrade is available. Library managers may prefer the Web client because it is more easily distributed, is customized, and provides a platform-free environment. Online searchers will seek out the interface that is most compatible with their computing capabilities and individual informationseeking styles. This may help the searcher achieve better results.

This article examines the impact of the system interface on searcher behavior and the quality of search results. A widely held assumption is that Web-based interfaces are easier to use. This is one reason for their

Steven J. Bell is director of the Paul J. Gutman Library at Philadelphia University; e-mail: bells@philau.edu. The author would like to gratefully acknowledge Dr. Michael Halperin for his assistance with the analysis and interpretation of the data; Jane Bryan for her helpful review and comments; and Jordana M. Y. Shane for her assistance with data collection and interviewing study participants. 


\section{Understanding Preferences for Search System Interfaces 441}

growing popularity as both the OPAC interface of choice and the interface most commercial database providers are adopting. Owing to an easier-to-interpret layout, contextual help at the click of the mouse, screens that guide searchers through the misunderstood world of boolean operators, and on-screen assistance, the Web client is becoming the interface that most library administrators are choosing to deliver over their networks. The fact that these administrators are choosing to "Webify" traditional library search systems raises the question of what real impact the evolution of the Web is having on the end user's ability to perform high-quality database searches.

This study examined how Web interfaces impact the quality or outcome of online searches, as well as the information-seeking behavior of the searcher.

A related issue addressed in this article is the challenge of conducting research on information-seeking behavior in digital environments. The difficulty lies in isolating the factor or factors responsible for a change in information-seeking behavior owing to the overwhelming number of variables that impact search behavior and outcomes. Creating a controlled environment that allows the researcher to isolate and identify those behavior-modifying variables is a research challenge. Consider just a few of these variables:

- general experience searching electronic data banks;

- specific knowledge of the data bank system;

- computer skill level (e.g., familiarity with GUls);

- system intricacies (e.g., search syntax and structural differences);

- individual willingness to experiment or take risks.

The difficulty in performing research on topics such as information-seeking behavior or user responses to search interfaces lies in controlling for the above variables. If not properly devised, the research methodology can mistakenly associate cause and effect with the wrong variable.

\section{Emotive Aspects of Searching}

A body of research has identified three important behaviors that have an impact on searching outcomes: cognitive, sensorimotor, and affective. Most of the information on these behaviors is based on research conducted by Diane Nahl and Carol Tenopir. ${ }^{1-3}$ Their studies of the search sessions of novice end users have indicated that search instructors place great emphasis on the cognitive and sensorimotor skills but rarely consider the impact of the emotive side of searching. Cognitive skills focus on the ability to understand search syntax and to use it to build search strategies; sensorimotor skills focus on the ability to understand what keys to use to make the system respond.

What is less understood, but equally critical to searching success, are the affective or emotional elements of searching. Affective elements determine how a searcher reacts to the search system. Levels of stress, frustration, anger, or surprise can affect an individual searcher's motivation to work through a difficult search or influence his or her decision to seek out alternate sources. A core finding of this body of research is that emotive aspects of searching significantly affect how people search and their level of satisfaction with their search results. Web-based interfaces are not a reaction to the need for systems that better respond to the realm of affective search behaviors, but an unanticipated outcome of their arrival may be a new era of interfaces that offer a search environment that satisfies the emotive needs of online database searchers. End users are typically frustrated by the arcane syntax structure of search systems traditionally designed for professional searchers. The technical knowledge required of searchers to use these systems tends to exacerbate the negative emotions connected to search behavior.

Public service librarians in academic libraries are familiar with end users who 
experience these emotional states. Having little or no formal online training, these searchers struggle with search systems such as DIALOG (through the Classroom Instruction Program) or Lexis-Nexis. And without a good deal of hand-holding from a professional searcher, these searches were usually dismal experiences with poor-quality results. With little understanding of the intricacies of these systems, most end users would have a negative search experience. How much of that is the fault of the traditional DOS or telnet interfaces used to search these systems, and how might the newer Web-based interfaces respond more appropriately to the emotive aspects of searching?

\section{Research Goals}

This study examined how Web interfaces impact the quality or outcome of online searches, as well as the informationseeking behavior of the searcher. Particular attention is paid to how two different search interfaces, telnet and Web, work to noticeably increase or decrease the impact of the emotive aspects of search behavior. Based on this author's experience as an instructor of online searching at a major library and information science program and in assisting many end-user searchers in academic library settings, an agitated emotional state rarely helps the vast majority of online searchers. Students of online searching who are known to be technically proficient with the cognitive and sensorimotor skills often fail to produce good results owing to the emotive aspects of searching (e.g., stress, lack of confidence, fear of failure).

Since the introduction of Web interfaces, the author has anecdotally observed a difference in the search behavior of some students. The Web interface provides what might best be described as a calming effect. With its more intuitive design, on-screen and contextual help at the click of a button, and in some cases the elimination of the "ticking clock" environment of traditional, payas-you go systems, the Web is a more sup- portive search environment. A goal of this study is to explore ways to more methodically identify exactly what impact Web-based interfaces have on the quality of online searching and the emotive aspects of search behavior. Stated as a hypothesis, online searching performed with Web-based interfaces allows for better search outcomes owing to the more supportive emotional environment provided for the searcher. Given a choice, searchers will seek out Web-based interfaces.

\section{Review of the Literature}

The Web-based interface is a relevant topic for a study of the impact that search system interfaces have on online searchers and their search outcomes. A review of the literature, using online searches of ERIC, LISA, Information Science Abstracts, $\mathrm{ABI} /$ Inform, Computer Database, and Education Abstracts Plus Full Text, was conducted by the author. No existing studies were found that exclusively examine how the introduction of Web interfaces has affected the quality of database searches conducted by either professional or end-user searchers. The existing literature on interfaces and database searching tends to concentrate on two areas-interface design issues, and examinations of the features of Web interfaces.

One article in particular examines the relationship between interface design and user ability to navigate the system more successfully. Karen Eliasen and colleagues studied the effect of terminology and screen layout on the ability of students to select databases from an online interface. Their study found that student search success increased as a result of enhancements to the database interface. ${ }^{4}$ However, the interface discussed in this article was not Web based. In an article that examined and compared the GUIs of several online database services, Allison J. Head focused on the elements of interface design and offered some insights (but no research data) into the connection between design and the ability of users to search the interface. $^{5}$ 


\section{Understanding Preferences for Search System Interfaces 443}

The interface design literature tends to discuss those features that contribute to, or detract from, a high-quality interface. Many of these articles are found in the literature of information science and instructional technology. Typical topics include screen layouts, menu structures, graphics and sounds, placement of controls, use of icons, and instruction screens. A good example is an article by Brian Vickery and Alina Vickery on online search interface design. This article suggested that design requirements must be set to provide maximum search assistance to inexperienced users. ${ }^{6}$ Other examples are targeted to interface designers rather than information retrieval specialists. A. P. Suess's article on interface design is a good example of an overview article. It covered the components of interface design, navigation systems, and things to consider when designing an interface. ${ }^{7} \mathrm{~A}$ number of articles are similar to Suess's, and there are dozens more on interface design. Although many of them discuss the impact of design on user ability to navigate or even learn from online systems, none have surfaced that connect the design of the interface to the quality of an online searcher's search results.

\section{Research Design}

The research design challenge was to create a study environment in which the differences between interfaces and their behavioral impact could be identified. This meant a design that eliminates, as much as possible, the influence of the numerous variables that affect search outcomes. To create a controlled environment for studying search behavior and outcomes, the author designed a study involving graduate students in an advanced online searching class. The students were members of a ten-week online searching class that offers five weeks of introductory searching and five weeks of advanced technique instruction. The course concentrates on the DIALOG system. Students learn the native mode search language of DIALOG, and the bulk of the instruction, class exercises, and assignments is done using a telnet client. Students spend part of one class session learning to use the DIALOG Web interface.

The participants in this study are hardly good examples of the typical end users most academic librarians confront in their daily patron interactions. These participants are using complex searching systems, performing far more difficult searches, and getting professional training. This study is designed to better understand why any searcher, professional or library patron, makes a particular interface choice and how that choice affects the quality of a search. In that respect, discoveries about motivational factors for interface choice and impact on search quality should be subject to generalizations from newly trained professional searchers to end-user populations. Both groups seek out search interfaces that allow the highest-quality outcome with the least chance for search failure. Despite the possibility that findings based on the experience of graduate online searching students may yield limited insight into the search behavior of library patrons, the online search class offers an opportunity to study search behavior for telnet and Web clients in a tightly controlled environment. A goal of this study was to determine if a similar search experiment might be possible with typical academic library end users.

Sixteen students from two different classes participated in this study. This number is not too small for the results to have statistical significance, but the differences in the sample results would have to be large to be significant. This study is as much a test of the research methodology as it is of whether Web interfaces can actually benefit searchers and improve their search results. Part of the exploration of methodology is the determination of a reliable method to gather data on searcher behavior. A questionnaire completed after searching, as well as an analysis of the actual search results, provided the study data. Six academic librarians participated in a parallel study. In addition to completing the questionnaire, the 


\section{College \& Research Libraries}

professional searchers were interviewed. The idea was to determine whether more insight could be gained from allowing the searchers to speak about their experience using both telnet and Web interfaces, immediately after the search sessions. However, for both groups, students and professional searchers, the design of the study was similar.

The main control element in this study is the use of DIALOG native search mode. DIALOG is unique in that both the telnet and Web interfaces can be searched using the native mode search language. Web-based search systems designed for end users typically feature a far simpler search apparatus with limited functionality that is easier to use than traditional search systems. To isolate the impact of the interface and determine user preferences for an interface type, it is crucial to eliminate the impact of the "ease-of-use" factor. That is, searchers might prefer one interface simply because it requires less effort to learn and use. In an experiment making use of DIALOG via telnet and Web in which both interfaces involve similar degrees of complexity in the use of search language and technique, it is possible to reduce the ease-of-use factor considerably. Participants needed to focus their comparison of the telnet and Web interfaces on factors such as degree of stress, time pressure, level of confidence, navigation, search speed, and information capture, rather than simply finding one interface easier to use than the other.

Another element of control is the use of online searching students as the study population. The participants are novice searchers. After ten weeks of training, all have roughly the same search experience and skill level. Being new to online search systems and interfaces, they are less likely than experienced searchers, or even a diversified group of end users, to have developed strong preferences for a particular interface and are more likely to be open to experimentation with different interfaces. Experienced searchers bring more system knowledge, greater famil-
September 1999

iarity with search techniques, and related expertise, all of which increase the difficulty of isolating the interface factors that influence search quality and searcher interface preference.

The actual experiment took the form of an in-class exam. Students were given three questions, DIALOG was used, and each question -involved at least two advanced DIALOG techniques. The questions were:

- Identify databases that contain full-text journals covering personal computing, then choose the top three databases and find the most frequently used descriptors for the Year 2000 crisis in those databases.

- Create a list of the top ten Japanese-owned banks in the United Kingdom, ranked according to total number of employees.

- OneSearch three files to find the three latest articles for a specific author, then determine how many times each of those articles has been cited and identify the author's most highly cited article ever published.

These questions are difficult to search correctly. They require the use of DIALOG techniques such as sorting and reporting; RANK; citation searching; duplicate detection; name finder files and the special reporting features of those files; file ranking, plus a strong familiarity with prefix search; and EXPAND techniques. To add to the challenge, students had only two hours to complete the exam.

The participants were required to use a specified interface for questions one and two. For example, for question one, one-half of the students had to use telnet and the other half had to use the Web. A student who had to use telnet for the first question was then required to use the Web for the second question. This design facilitated a comparison of the search results to determine whether any difference in their quality could be attributed to the interface used. All of the telnet search sessions from both questions one and two could be closely compared to all of the Web search sessions for the same ques- 


\section{Understanding Preferences for Search System Interfaces 445}

tion. For the third question, participants were free to choose either interface. This provided some insight into participant preference for an interface.

All search sessions were captured to disc so that the entire search session would be available for review. This can be done easily with the telnet searches, but continuous capture of a search session is not possible on the Web. For the Web searches, participants could only capture specific Web pages to disc, such as the page with search results, the page that provides the search strategy summary (result of a "display sets" command), and the cost/time display. To supplement recorded search sessions, students also submitted, in writing, their search statements, database choices, and other explanatory comments.

At the end of the exam, students were given a questionnaire. The questions asked participants to compare or rate the telnet and Web interfaces in several categories, and were designed to collect information on their attitudes toward and preferences for each interface. Completing the questionnaire was voluntary, and students were informed that the questionnaires would be reviewed only after submission of the final grades. Of the sixteen participants, eleven returned the questionnaire. The professional participants completed six additional questionnaires.

\section{Analysis of the Searches and Questionnaires}

Because data collection was not the result of a random sample but, in fact, resulted from a self-selected sample, the data do not meet the necessary standards for formal statistical testing. Data collection took place under fairly stressful circumstances (an exam environment), and the number of subjects was small. In an ideal situation, several hypotheses would be tested, such as:

- The Web environment is more conducive to successful search outcomes than the telnet environment; searchers will also find the web interface easier to use.

- Web searchers will have a higher level of perceived search success.
- The Web interface provides a lowerstress search environment.

- Searchers will prefer the Web mode for future searching.

Although these hypotheses are not tested statistically, a review of the searches conducted by the participants and the questionnaire responses offers a fair amount of anecdotal evidence. Despite the results reflecting the work of a small group of library program students at exam time, this evidence can be used (because there is little else on this subject) to initiate discussions of the impact of Web interfaces on users of the Web-based library databases.

The data analysis leads the author to conclude there is no conclusive evidence to support a hypothesis that Web interfaces contribute to better search outcomes owing to a more "searcher-friendly" environment. The survey results do indicate that the searchers found the Web environment less stressful than the telnet environment. Although the Web may be somewhat effective at neutralizing some negative emotive aspects of online searching, it appears that other, more influential factors determine what interface a searcher will choose. The findings of this study do provide some insight into searcher preferences for interfaces, and one of the more revealing results suggests that the relation of a system interface to searcher success may be less critical than previously thought.

A direct comparison of each searcher's Web and telnet searches reveals that any difference in search quality was negligible. Searchers who performed well using telnet, performed equally well using the Web interface. The most critical determinant of search success appears to be how well the searcher knows and understands the search system. In DIALOG searching, no interface can help a poorly trained or ill-prepared searcher, whether a novice end user or a professional searcher. If the searcher knows what to do and how to do it, the search will be successful in any interface environment. However, the Web environment can pro- 


\section{College \& Research Libraries}

vide an advantage to a searcher with weaker skills or one searching in a less familiar system.

For example, one of the searches required the use of a directory database the students had never used before. To perform the search correctly, students had to find three required prefix search codes. The Web environment facilitates this part of the search process by providing quick access to the codes from the same screen where the search strategy is being formulated. Telnet searching would require more effort to obtain the same code information. The same search also required the searcher to compile a report using the DIALOG sort and report commands. Searchers who did not know to use these commands or how to execute them properly would get poor results regardless of the search interface.

Other indicators support the observation that search interface is less critical to searcher success than hypothesized. Respondents were asked to rank-order several factors that influenced their search success. The highest-rated factor, as shown in table 1, was "familiarity with techniques required to perform a search correctly." This factor was ranked 1 or 2 by 65 percent of the respondents. Online search instructors know that students do well when they achieve a comfort level with a particular technique or system. When asked to perform the same technique in a less-well-known system or to perform a new function based on a slight variation on a familiar technique, many students experience difficulty. Given a choice of systems to perform a similar search, most students and professional
September 1999

searchers prefer the system they first learned-in other words, the system with which they are most familiar.

Table I further shows that search interface is less important to achieving highquality results. Seventy-three percent of the respondents rated the factor "search mode used to perform the search" fifth out of five possible influential factors on search success. What could be termed as more "practical" searcher concerns are consistently ranked higher than search mode. The nature of the question (particularly its level of difficulty), the amount of time allowed for the question, and even the availability of search documentation (students could use any notes or other documentation gathered during the semester for the exam) are all ranked above search interface.

One common characteristic among the factors that ranked above search interface is that all are relatively tangible. The rankings may be influenced by the searcher being able to readily point to factors such as specific techniques or amount of time to complete a search as having an impact on their results. It may be more difficult to gauge the impact of an interface. Screen design is a less tangible feature of the search session. It tends to be noticed only when there is a technical problem. Consider a simple, but significant, difference in the telnet and Web modes-the correction of typing errors. This is considerably easier with a Web interface. Using telnet, a searcher with a typing error in the second word of a seven-word statement would need to backspace over the entire statement to get to the error and then retype the statement.

\begin{tabular}{|cl|}
\hline & \multicolumn{1}{c|}{ TABLE 1} \\
& Respondents' Ranking of Factors That Influence Search Success \\
\hline \hline Rank & Factor Description \\
\hline 1 & Familiarity with techniques required to perform the search correctly \\
2 & Nature and difficulty level of the search question \\
3 & Amount of time allowed to perform the search \\
4 & Documentation available at the time of the search \\
5 & Search mode used to perform the search \\
\hline
\end{tabular}


Understanding Preferences for Search System Interfaces 447

\begin{tabular}{|lcc|}
\hline \multicolumn{3}{|c|}{$\begin{array}{c}\text { TABLE 2 } \\
\text { Respondent-Perceived } \\
\text { Stress Level }\end{array}$} \\
\hline \hline Mode & High Stress & Low Stress \\
\hline Telnet & $73 \%$ & $27 \%$ \\
Web & $36 \%$ & $64 \%$ \\
\hline
\end{tabular}

In the Web mode, the searcher could simply put the insert tool where the error is, make the correction, and then continue the search.

These less-noticeable features can save time and prevent frustration during search sessions. When asked to rank the importance of interface to search quality, participants may have overlooked these simple differences in the search modes. This question relates to the larger issue raised in this study of the impact of research design. A research design could be structured to place greater emphasis on the impact of search mode to the quality of the search session than the design used in this study does. But the researcher would need to find a way to get the participants to pay greater attention to the intangible elements of the search session than to the more obvious, tangible factors. Alternately, the use of postsearch interviews could better probe searchers about these factors.

A researcher also might question the viability of the research design because of the inconsistencies that surface in the analysis of participant responses. This study's only consistency might be the regular inconsistency of its findings. Tables 2,3 , and 4 reflect the objectives of the design to get participants to indicate their preferences for a specific mode in a more quantifiable fashion. A researcher might be able to reach some conclusions about searcher interface preferences from tables 2 and 3 , but table 4 immediately shatters any effort to support a specific conclusion. Table 2 provides evidence that

\begin{tabular}{|lcc|}
\hline \multicolumn{3}{|c|}{$\begin{array}{c}\text { TABLE 3 } \\
\text { Reported Degree of Search } \\
\text { Ease by Mode }\end{array}$} \\
\hline \hline Mode & Easy & Difficult \\
\hline Telnet & $64 \%$ & $36 \%$ \\
Web & $36 \%$ & $64 \%$ \\
\hline
\end{tabular}

the Web environment does indeed cater to the emotive elements of the search process by providing a less-stressful search level. Stress can lead to increased pressure, which in turn can lead to search errors, both simple and complex. That might lead a researcher to expect that participants would find their search sessions easier in the Web environment, but the results in table 3 suggest that the respondents strongly favored the telnet mode for its greater ease of use.

When the participants were asked which search mode leads to search success, the findings are again inconsistent. Table 4 shows that the searchers rated their degree of success with both the Web and telnet modes nearly equal. In other words, despite their clear preferences for the Web or telnet modes in tables 2 and 3 , in table 4 the participants show no strong perception that one mode or the other helped to achieve greater search success. Findings such as these make it difficult for the researcher to formulate solid conclusions. However, the results of table 4 do appear somewhat consistent with the review of the actual searches. That is, the searches themselves are not consistently more successful in one mode or the other. They are equally successful in both modes, depending more on whether the searcher knew what to do achieve search

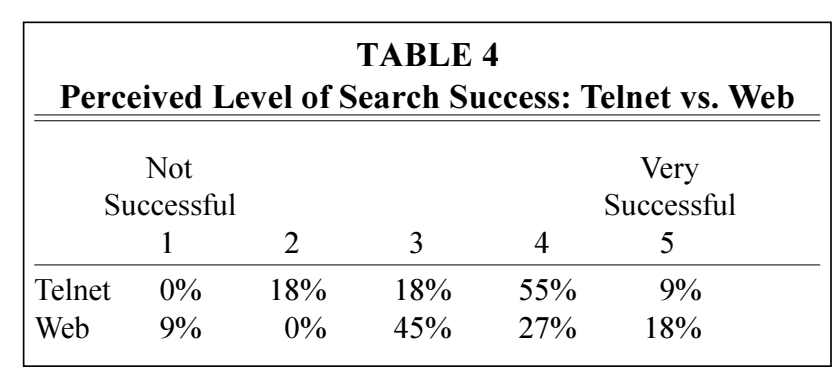


448 College \& Research Libraries

\begin{tabular}{|lcc|}
\hline \multicolumn{3}{|c|}{$\begin{array}{c}\text { TABLE 5 } \\
\text { Searcher Preference for Future } \\
\text { Searching }\end{array}$} \\
\hline \hline Mode & Prefer to use & $\begin{array}{c}\text { Prefer Not } \\
\text { to Use }\end{array}$ \\
& & $50 \%$ \\
Telnet & $50 \%$ & $50 \%$ \\
Web & $50 \%$ & \\
\hline
\end{tabular}

success. Therefore, it may be reasonable to expect the searchers to have no strong perception that they achieve success in one mode over another.

Some additional inconsistency is evidenced in the data provided in tables 5 and 6 . According to table 5, the participants show no clear preference for either mode for their future searching needs. However, according to table 6, when the participants were able to express a preference for a specific mode, they overwhelmingly showed a preference for telnet mode for the exam's third search question. If there is such a strong preference for telnet, why, then, is it not the clear choice for future searching? An explanation is suggested by the responses of the professional searchers who were interviewed after they had done searches in both modes. This type of inconsistent response may be attributed to risk factors and a searcher's personal tendencies to accept or avoid risk. Some of the professional searchers indicated their willingness to experiment with the new Web interface. Others indicated they would prefer, given the importance of getting good search results, to stay with telnet because they were comfortable with it and had used it successfully in the past.

That attitude appears to be mirrored in tables 5 and 6 . In a testlike situation, where good results are critical, searchers are less likely to take a risk on a new, lesser-known entity. The majority selection of telnet for the third search suggests that the participants, having spent the bulk of
September 1999

\begin{tabular}{|lc|}
\hline \multicolumn{3}{|c|}{$\begin{array}{c}\text { TABLE 6 } \\
\text { Search Mode Selected for } \\
\text { Third Search }\end{array}$} \\
\hline \hline Mode & Percent Selecting Mode for \\
& Third Search \\
\hline Telnet & $82 \%$ \\
Web & $18 \%$ \\
\hline
\end{tabular}

their in-class and home search time using telnet, were unwilling to take the risk of abandoning telnet under exam circumstances. With their overall attitudes toward online searching and interface options, the newly trained searchers (given their responses in table 5) appear more willing to try the newer interface in the future than experienced searchers are. The professional searchers were less willing to work with a new Web interface but indicated that the Web environment was insufficiently robust at this time to provide the proper search tools. Most did add that they believe future Web interfaces will work much more efficiently than they do now and that they would make use of the Web for online searching when they see improvement. Interpreted another way, the Web will be a better option for professional searchers when interface developers eliminate the current risk factors or are able to make the Web work more like telnet.

Choosing between telnet and Web modes is more than a matter of prefer-

\begin{tabular}{|lcc|}
\hline \multicolumn{3}{|c|}{$\begin{array}{c}\text { TABLE 7 } \\
\text { Searcher Ratings of Factors That } \\
\text { Influence Mode Preference }\end{array}$} \\
\hline \hline Factor & Telnet & Web \\
\hline Stress & High & Low \\
Time pressure & High & Low \\
Familiarity & More & Less \\
Data capture & Better & Worse \\
Search speed & Better & Worse \\
System help & Low & High \\
Displays & High & Low \\
User friendly & Low & High \\
Navigation & Clear & Ambiguous \\
\hline
\end{tabular}




\section{Understanding Preferences for Search System Interfaces 449}

ring a character-based interface or a GUI. Certain practical concerns give one mode advantages over the other for online searching. Table 7 provides some insight into what those practical considerations are. Although the ratings reinforce the Web mode as the superior option for easing the negative emotive forces of the online search environment, telnet rates higher in those areas where searchers rely on an interface to help provide fast and efficient sessions. Foremost among these factors are speed and data capture. Even though the time pressure is higher in the telnet environment, searchers want to get the job done quickly. The professional searchers uniformly were displeased with waiting for the Web to respond to queries and with the extra time needed to navigate through record displays.

Scarce attention is given to other forces, such as stress, frustration, anger, or surprise, that affect a searcher's emotional state and influence the outcome of a search session.

A significant weakness of the Web interface is the handling of data capture. Searchers still prefer the ability to continuously download search sessions and record displays. This is not possible in the Web environment. Individual pages must be downloaded, and this can be inadequate for a searcher who wants the complete session. The interviews with professional searchers indicate that this is a significant factor in determining their preference for the telnet mode. The Web environment may appeal to the searcher's emotive side, but practical considerations are strong determinants of preferences for an interface. Despite the negative emotive forces of the telnet mode, owing to searchers' familiarity with it and the "not-quite-ready- for -prime-time" feel of most commercial Web search products, the ultimate inconsistency is that telnet mode is preferred despite the more searcher-friendly Web environment.

\section{Summary of Analysis and Findings}

This study sought to develop a better understanding of online searcher preference for a search interface, the factors that influence searcher preferences, and how an interface impacts the quality of search results. Traditional online search instruction, at both the professional and end-user level, emphasizes the cognitive and sensorimotor skills required for search success. Scarce attention is given to other forces, such as stress, frustration, anger, or surprise, that affect a searcher's emotional state and influence the outcome of a search session. Traditional search interfaces, including DOS and telnet systems that are primarily command driven and time sensitive, are known to contribute to the pressure, tension, and stress experienced by online searchers. These negative emotive forces tend to contribute to unpleasant search experiences that result in unsatisfactory outcomes.

It is the very nature of the Web search environment, the author hypothesized, that would allow newly trained searchers and experienced professionals to improve the quality of their searches. Relieved from the pressures of the "ticking meter" syndrome and supported with devices such as hypertext links to documentation, the author believed that searchers would be better able to concentrate on the cognitive and sensorimotor elements of searching. However, the analyzed search results failed to support the hypothesis. No significant difference in search quality was detected when directly comparing searches performed with both telnet and Web interfaces. Rather, search quality was determined by a searcher's understanding of the search question, familiarity with the techniques required to perform the search correctly, and knowledge of the search system's syntax and structure. When asked to state their interface preferences, despite the searchers' higher comfort level with Web interfaces, for largely practical reasons, most preferred telnet-mode searching.

The demonstrated preference for character-based interfaces in this study in 


\section{College \& Research Libraries}

no way suggests that a rethinking of database producers' migration to Web interfaces is in order. That trend, in this author's opinion, has yielded mostly positive benefits for library administrators, frontline public service librarians, and, most important, library users. A reversal of this migration to Web interfaces would be irrational given the benefits of the current Web-based search environment. Hypertext, platform freedom, simpler-by-comparison syntax, guided searching, search redirection via hypertext linking, and GUIs provide a rich search environment that is far better understood by end users. The Web environment also encourages end users to experiment, take risks, and explore uncharted territories. To add to their advantages, Web-based systems are now introducing alternate search engines, such as relevancy retrieval systems, that further aid end users who have little knowledge of the rules of keyword searching or arcane search syntax.

Much of the study participants' preference for telnet-mode searching is based on those practical benefits known to professional searchers for decades. Those features have yet to migrate to the Web environment. The response of online searchers to this study's findings should be to urge database producers to build the strengths of character-based interfaces into their Web designs. Pressure can be brought on the developers of Web interfaces to provide systems that are optimized for search speed and data capture. Many enhancements and innovations in Web-based search systems result directly from customer suggestions. The tremendous progress of the past few years gives great reason to believe that Web interfaces will continue to improve.

\section{Conclusion}

Academic librarians teach end users to be wise consumers of electronic information. College and university students should be information literate. Web-based interfaces can further a librarian's ability to help students find needed information

\section{September 1999}

and support our goal of raising our students' information literacy proficiency. But the surface simplicity of Web interfaces should not lull the profession into a retreat from traditional instructional efforts. If anything, the findings of this experimental research reinforce the importance of end-user training programs. Quality search outcomes, as this study shows, are still largely dependent on basic knowledge of search system features, familiarity with the search techniques required to obtain certain kinds of information, and the acquisition of a comfort level with a database system. An interface alone, although it may provide a more or less supportive search environment, fails to guarantee successful search outcomes.

To further the goals of helping students achieve information literacy in an environment dominated by Web-based interfaces, in which end users are more likely than ever to assume that Web-based searching is simple, members of our profession should consider emphasizing the following factors in their outreach to end users:

- Teach search skills. Although the importance of teaching traditional boolean logic may diminish, the value of learning how search engines work, why systems offer special features such as limiting, and techniques for capturing and postprocessing retrieved information will increase. Bibliographic instruction should change accordingly to respond to new needs, concentrating on contemporary issues, such as the importance of evaluating information found on the Web, or simply teaching students to distinguish between free information on the Web and information found in the library's commercial online databases.

- Familiarity influences search behavior. End users will be reluctant to accept new systems and will favor old ones. They take a "loss perspective" when an old system changes or is replaced. They focus on what is lost. The emphasis for new systems must be placed on what is gained. When introducing new systems, take steps to encourage the growth of familiarity and allow adequate time for user 


\section{Understanding Preferences for Search System Interfaces 451}

acceptance to grow. As above, bibliographic instruction will foster acceptance but so, too, do marketing efforts such as library events geared to introducing and popularizing specific search systems.

- Practical considerations influence search behavior and system choice. End users will choose the search system that optimizes their practical needs. If a full-text article is needed, a full-text system will be chosen over a better system offering only partial or no full text. A system that allows free printing or e-mail will be chosen over a system that offers neither. In developing search skills and system familiarity, appeal to the end users' sense of what offers practical value.

- Work independently with consortium partners and system vendors to optimize Web interface design, emphasizing the need for easy-to-use tutorials, quick search tips, and clear descriptions of resources.

Web-based interfaces are likely to dominate the landscape of commercial library database systems. Even so, it is probably too soon to herald the demise of telnet and Windows-based interfaces. If anything, the landscape may grow more tangled as the deployment of java-based interfaces, combining elements of the Web's ease of distribution and Windows's speed and functionality, become more predominant. The exploration of interfaces and their impact on information-seeking behavior, the quality of search sessions, and the emotive elements of online searching should continue to be a rich field for study. Determining how to best conduct this research will continue to be a challenge and will no doubt return to the core issue of how to isolate the interface amidst all of the variables that contribute to the outcome of an online search experience.
Though only partially successful, the research design used in this study may provide some insights into developing effective methods for studying the impact of interface design on end-user searching. Future research designs must consider methods of eliminating the natural bias toward familiar or first-learned interfaces. For example, a study could select participants based on their degree of online searching experience. An experiment that included only participants without any online search experience could potentially eliminate any bias factors. Study groups could be exposed to different interfaces in equal parts to decrease the likelihood of creating bias toward a specific interface. A totally different tact might be to expose new searchers to just one interface and then compare their search results to those experiencing another interface. Data collection techniques could combine several methods, including questionnaires and interviews both during and after search sessions. In this study, both methods yielded beneficial insights into information-seeking behavior.

Whatever research design or study methods are used, researchers should continue to explore and study search interfaces and their impact on online searching. Our profession must make a commitment to increase the efficiency and effectiveness of our own searching abilities in order to help the next generation of professionals achieve success as searchers and to create search environments that help our users successfully connect to needed information. That makes it our responsibility to identify the features of interfaces that further these goals and to communicate this knowledge to the database producers who will create the new generation of products that help us do our job.

\section{Notes}

1. Carol Tenopir, "The Emotions of Searching," Library Journal 119 (Sept. 1994): 134-35.

2. Carol Tenopir, Diane Nahl-Jakobovits, and Dara Lee Howard, "Strategies and Assessments Online: Novices' Experience," Library and Information Science Research 13 (July-Sept. 1991): 23766. 
3. Diane Nahl, and Carol Tenopir, "Affective and Cognitive Searching Behavior of Novice End-Users of a Full-Text Database," Journal of the American Society for Information Science 47 (Apr. 1996): 276-86.

4. Karen Eliasen, Jill McKinstry, Beth M. Fraser, and Elizabeth P. Babbitt, “Navigating Online Menus: A Quantitative Experiment," College \& Research Libraries 58 (Nov. 1997): 509-16.

5. Alison J. Head, "A Question of Interface Design: How Do Online Service GUIs Measure Up?" Online 21 (May-June 1997): 20-24, 26-29.

6. Brian Vickery and Alina Vickery, "Online Search Interface Design," Journal of Documentation 49 (June 1993): 103-87.

7. A. P. Suess, "Designing the User Interface," Journal of Interactive Instruction Development 10 (fall 1997): 28-33. 